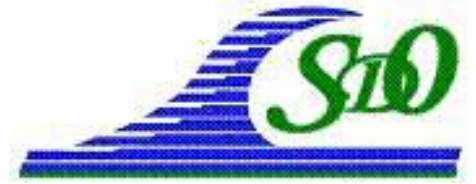

\title{
Modélisation physique de la morphodynamique d'une plage barrée tridimensionnelle
}

\author{
Hervé MICHALLET ${ }^{1}$, Bruno CASTELLE ${ }^{2}$, Frédéric BOUCHETTE ${ }^{3}$, \\ Adrien LAMBERT ${ }^{3}$, Céline BERNI ${ }^{1}$, Eric BARTHELEMY ${ }^{1}$, \\ Philippe BONNETON ${ }^{2}$, Damien SOUS ${ }^{4}$
}

1. LEGI (UMR 5519 CNRS, Université Grenoble), BP53, 38041 Grenoble cedex, France.

herve.michallet@hmg.inpg.fr

2. UMR CNRS 5805 EPOC, Université de Bordeaux, Avenue des Facultés, 33405 Talence Cedex, France.

3. Institut de Mathématiques et de Modélisation/Géosciences-M, Univ. Montpellier 2, Place E. Bataillon, 34095 Montpellier cedex 5, France.

4. LSEET, Université de Toulon et du Var, 83957 La Garde Cedex, France.

\section{Résumé :}

Des expériences dans un bassin de $30 \mathrm{~m}$ × $30 \mathrm{~m}$ ont été réalisées dans l'objectif de caractériser la dynamique d'un courant d'arrachement et les évolutions morphologiques associées. La plage de sable fin (diamètre médian 166 microns) couvrait la largeur du bassin (30 m en longshore) pour un profil total de $20 \mathrm{~m}$ cross-shore. La formation de courants sagittaux a été forcée par une houle frontale (spectre de Jonswap) avec un déficit d'énergie au centre du bassin.

Une séquence accrétive complète de plage intermédiaire à simple barre (selon classification de WRIGHT \& SHORT, 1984) a été reproduite. La barre s'est progressivement tridimensionnalisée en forme de croissant puis s'est connectée au haut de plage avec la présence de deux chenaux profonds qui se sont ensuite comblés pour obtenir une plage à terrasse au bout de plusieurs dizaines d'heures. L'association des mesures hydrodynamiques et morphodynamiques montre que le comblement des chenaux est alimenté par le haut de plage.

\section{Mots-clés :}

Courant d'arrachement - Plage intermédiaire - Barre sableuse - Déferlement

\section{Introduction}

La formation et l'évolution, sur les plages de sable, de structures sédimentaires rythmiques de grandes dimensions restent encore mal comprises. La mise en place de courants d'arrachement, associés au creusement de chenaux de vidange, forme les alternances de barres et de baïnes fréquemment observées sur le littoral Aquitain. Ce type de courant, engendré par les variations longshore des tensions de radiation, est présent sur la plupart des littoraux suffisamment rectilignes (MACMAHAN et al., 
2006). Une bonne connaissance de ces courants est nécessaire pour sécuriser les zones de baignade mais également pour prédire correctement les évolutions du trait de côte, puisqu'ils contribuent à localiser l'érosion de la plage et de la dune durant les tempêtes. De nombreuses expériences de terrain ont été menées pour chercher à comprendre leur dynamique et des efforts importants de modélisation numérique sont poursuivis (e.g. BRUNEAU et al., 2009). En revanche, très peu d'expériences de laboratoire ont cherché à reproduire ce type de phénomène.
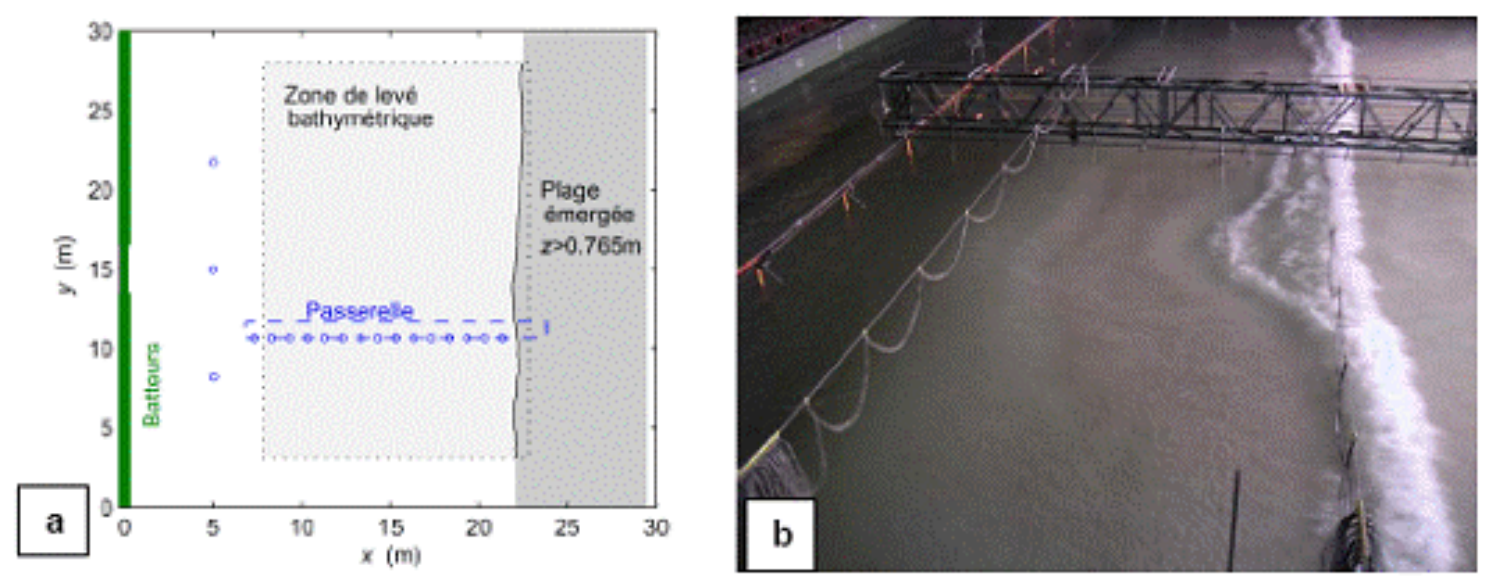

Figure 1. (a) Schéma du bassin (x cross-shore et y long-shore). La profondeur d'eau est $h_{0}=0.765 \mathrm{~m}$ pour $x<6 \mathrm{~m}$. La plage de sable s'étend de $x \approx 6 \mathrm{~m}$ jusqu'à $x=26 \mathrm{~m}$. La passerelle permet les relevés bathymétriques dans la zone grisée délimitée par le trait

en pointillés. Les cercles indiquent les sondes capacitives de mesure de niveau de surface libre (3 sondes fixes en entrée du domaine et 15 sondes placées sur la passerelle de levé permettant la mesure des vagues selon différents transects). (b) Image d'une vague déferlée, batteurs en haut à gauche, passerelle de levé supportant les sondes.

\section{Conditions d'expériences}

Des expériences dans le bassin de $30 \mathrm{~m}$ par $30 \mathrm{~m}$ du Laboratoire d'Hydraulique de France (G-INP - Sogréah) ont été réalisées dans l'objectif de caractériser la dynamique d'un courant d'arrachement et les évolutions morphologiques associées. Le dimensionnement des expériences considère une échelle de longueur de 1/10 qui, par similitude de Froude, impose une échelle de temps de l'ordre de 1/3. Pour reproduire correctement le transport sédimentaire en similitude, GRASSO et al. (2009) ont réalisé des expériences en canal avec un sédiment de faible densité (1.19). Pour une question de coût ce choix n'a pas pu être fait ici. Le sédiment utilisé est un sable de diamètre médian 166 microns (vitesse de chute $w_{s}=2.0 \mathrm{~cm} / \mathrm{s}$ ) qui permet de bien reproduire la dynamique du sédiment en suspension. En revanche, le nombre de Shields associé étant plus faible qu'en nature, le régime de transport en sheet flow n'est pas très bien reproduit dans cette expérience. 
Le dispositif expérimental est schématisé sur la figure 1a. La plage de sable couvrait la largeur du bassin (30 m en longshore) pour un profil total de $20 \mathrm{~m}$ cross-shore, sur une épaisseur d'environ 15 à $20 \mathrm{~cm}$ au-dessus d'un soubassement de gravier recouvert de géotextile. Une sonde laser placée sur une passerelle pilotée pour couvrir l'essentiel de la plage a permis de lever régulièrement les bathymétries. Cette technique de mesure nécessitait la vidange complète du bassin pour effectuer un levé. Le bassin était rempli chaque matin, suffisamment lentement pour ne pas perturber les fonds, jusqu'à atteindre une profondeur d'eau aux batteurs $h_{0}=0.765 \mathrm{~m}$.

Les expériences consistaient à générer des séquences de $20 \mathrm{mn}$ de houle de spectre de Jonswap caractérisée par une hauteur de vague significative $\left(H_{m 0}\right)$ et une période pic $\left(T_{p}\right)$. La mise en place de circulations a été forcée par une houle frontale avec un déficit d'énergie au centre du bassin en imposant diverses lois d'amortissement aux batteurs. La figure 2 donne un exemple de la variation de la hauteur de vague à l'attaque de la plage (profondeur d'eau d'environ $60 \mathrm{~cm}$ ) pour différentes lois d'amortissement.

Les sondes de mesures de vagues (sondes capacitives conçus par Sogréah) et de vitesses (ADV Vector de Nortek) ont été disposées principalement sur la passerelle de levé de fond ce qui a permis de caractériser les vagues et les vitesses induites selon différents transects. La passerelle était généralement déplacée à la fin de chaque séquence de 20 mn. De 7 à 26 séquences successives constituaient chacune des expériences. A l'issue de chaque expérience, le bassin était vidangé pour permettre de réaliser le levé bathymétrique pendant la nuit.

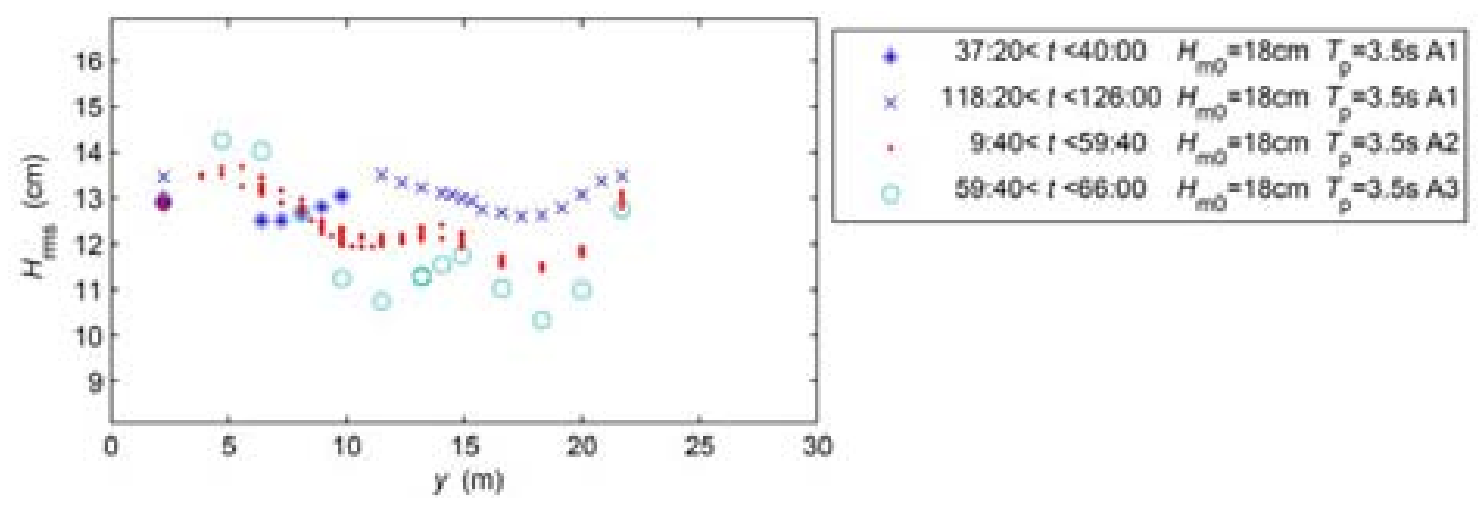

Figure 2. Variation longshore de la hauteur de vague quadratique moyenne mesurée en $x=7.3$ m pour différentes conditions d'amortissement des batteurs.

\section{Evolutions morphologiques}

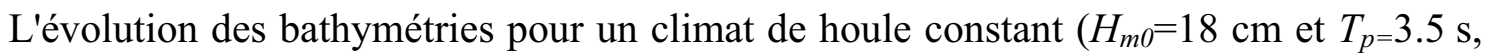
loi d'amortissement A2 sur la figure 2, à l'exception d'une courte séquence avec la loi A1 entre 37:20 et 40:00) est montrée sur la figure 3. Débutant par une plage quasiment uniforme en longshore ( $t=9: 40)$, une barre en croissant s'est progressivement formée $(t=15: 40)$ puis s'est connectée au haut de plage avec la présence de deux chenaux 
Thème 2 -Dynamique sédimentaire et transports des particules

profonds perpendiculaires à la côte ( $t=37: 20$ en $y \approx 10 \mathrm{~m}$ et $y \approx 22 \mathrm{~m})$ qui se sont ensuite comblés pour obtenir une plage à terrasse au bout de plusieurs dizaines d'heures. Cette séquence correspond à la première modélisation physique de la séquence accrétive complète des plages intermédiaires (le nombre de Dean $\Omega=H_{m 0} / T_{p} w_{s}$ est égal à 2.3) à simple barre suivant la classification de WRIGHT \& SHORT (1984). On observe en effet une morphologie de type Rhythmic Bar and Beach (RBB, $t=9: 40$ à $t=21: 00$ sur la figure 2) puis de type Transverse Bar an Rip (TBR, $t=31: 20$ à $t=45: 40$ ) et enfin de type Low Tide Terrace (LTT, $t=51: 40$ à $t=59: 40$ ).
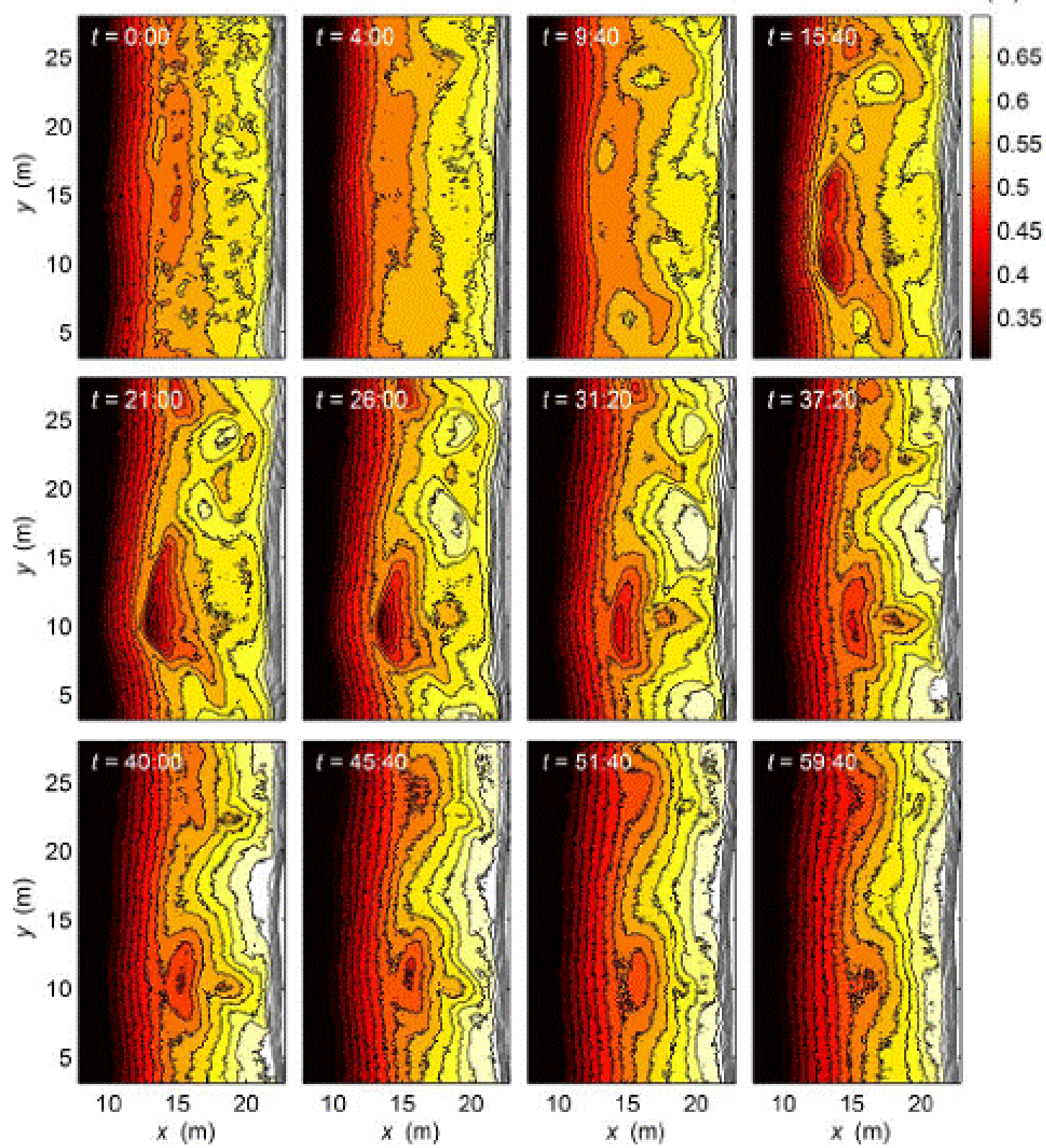

Figure 3. Evolution des bathymétries en phase accrétive pour $H_{m 0}=18 \mathrm{~cm}$ et $T_{p}=3.5 \mathrm{~s}$. 

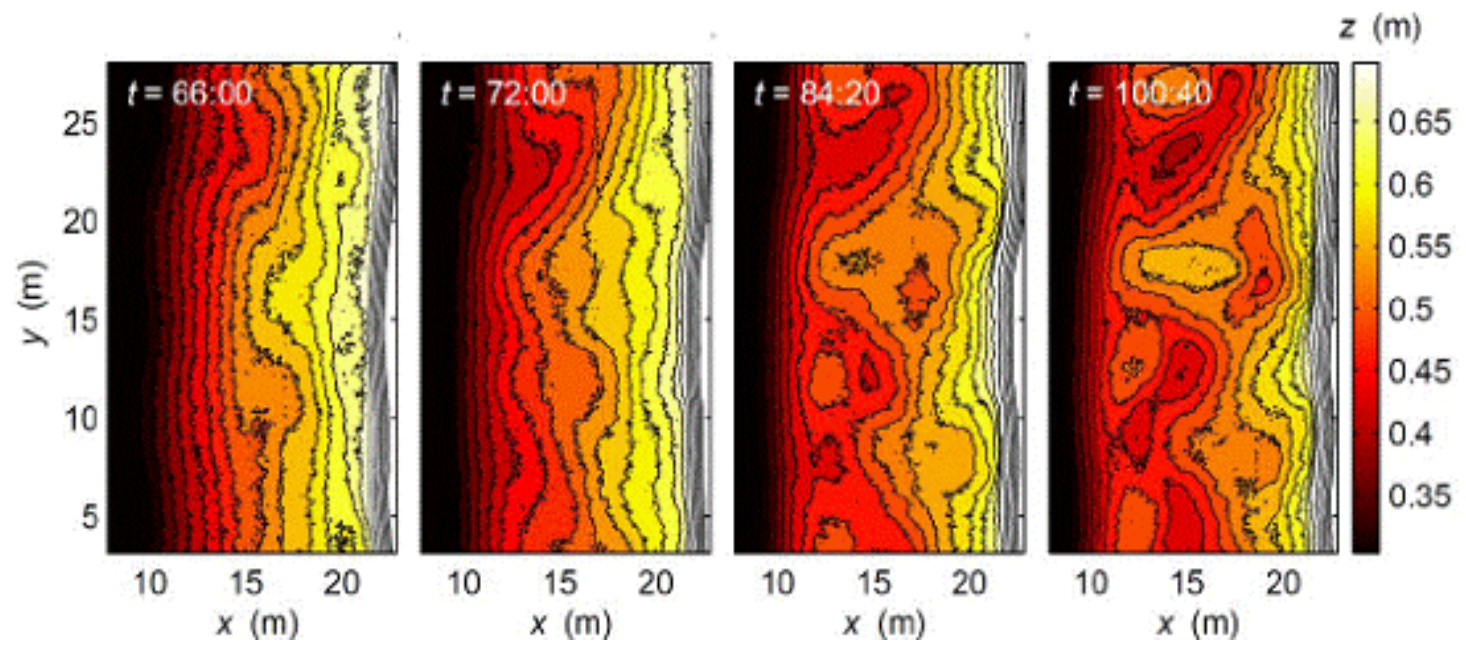

Figure 4. Evolution des bathymétries en phase érosive pour $H_{m 0}=23 \mathrm{~cm}$ et $T_{p}=2.3 \mathrm{~s}$.

Une séquence érosive pour un climat plus énergétique $\left(H_{m 0}=23 \mathrm{~cm}\right.$ et $T_{p}=2.3 \mathrm{~s}$, soit $\Omega=5.0$ qui indique une évolution vers une plage dissipative) est montrée sur la figure 4 . Au cours de cette évolution, la plage s'est fortement tridimensionnalisée sans privilégier la formation de chenaux perpendiculaires à la plage.

Les profils de plage moyennés en longshore sont montrés sur la figure 5a. L'évolution au fil des expériences de la hauteur moyenne de lit pour la partie supérieure de la plage $(x>14 \mathrm{~m})$ est montrée sur la figure $5 \mathrm{~b}$. Elle souligne que la tendance à l'accrétion n'est pas uniforme en longshore, avec une accrétion beaucoup plus forte au centre du bassin $(y=18.3 \mathrm{~m})$ et une érosion dans l'axe du creusement du chenal $(y=10.7 \mathrm{~m})$. Au cours de la séquence suivante ( $t \geq 66: 00)$, l'érosion globale est davantage similaire selon les différents transects.

Un paramètre de tridimensionnalité a été estimé par la formule :

$$
\sigma_{z}=\left\langle\sqrt{\frac{1}{y_{2}-y_{1}} \int_{y_{1}}^{y_{2}}(z(x, y)-\overline{z(x)})^{2} d x}\right\rangle
$$

où $z(x, y)$ est la cote du fond pour $7.8 \mathrm{~m}<x<22.8 \mathrm{~m}, y_{1}=3.12 \mathrm{~m}$ et $y_{2}=19 \mathrm{~m}$, et $\overline{z(x)}$ la moyenne longshore entre $y_{1}$ et $y_{2}$. La variation de ce paramètre au fil des expériences est tracée sur la figure $5 \mathrm{c}$. Elle confirme une forte augmentation de la tridimensionnalité de la plage lors de la formation de la barre en croissant puis des chenaux ; une diminution lors du comblement des chenaux et une réaugmentation durant la séquence érosive. CASTELLE et al. (2010) ont montré que l'intensité du courant dans le chenal est linéairement corrélé au paramètre $\sigma_{\mathrm{z}}$.

\section{Eléments de caractérisation de l'hydrodynamique}

Un exemple de l'évolution de la hauteur de vague quadratique moyenne le long de la plage est présenté figure 6. Les mesures en zone de levée (figure 6a) indiquent que le 
front d'onde incident, non-uniforme à l'attaque de la plage $(x=8.3 \mathrm{~m})$, se réfracte de façon complexe alors que la bathymétrie présente peu de variabilité longshore à ces profondeurs. Le raidissement des vagues est plus intense dans l'axe du chenal $(y \approx 10 \mathrm{~m}$, entre $x=8.3 \mathrm{~m}$ et $x=12.3 \mathrm{~m}$ ). On observe une diminution de $H_{\mathrm{rms}}$ entre $x=13.3 \mathrm{~m}$ et $x=14.3 \mathrm{~m}$ ce qui indique le début du déferlement. Le déferlement sur la barre est plus intense dans l'axe du chenal, il en résulte que la hauteur de vague est à peu près uniforme en longshore en $x=15.3 \mathrm{~m}$ (figure 6b). Le déferlement devient ensuite moins intense dans le chenal puisque la profondeur d'eau y est plus importante. Les vagues dissipent brutalement leur énergie en haut de plage pour $x \geq 19.3 \mathrm{~m}$.

Sur la figure 7 est présenté un exemple des variations longshore des vitesses Eulériennes au niveau de l'évasement du courant d'arrachement. Cet évasement est caractérisé par un courant de retour moyen qui atteint $10 \mathrm{~cm} / \mathrm{s}$ dans l'axe du chenal, tandis que les vitesses moyennes longshore sont orientées vers les $y$ négatifs pour $y \leq 10$ $\mathrm{m}$ et vers les $y$ positifs pour $y \geq 10 \mathrm{~m}$ (figure $7 \mathrm{a}$ ). A cette position cross-shore, les vitesses quadratiques moyennes sont moins fortes dans le chenal (figure $7 \mathrm{~b}$ ), ce qui indique un déferlement moins intense.

Ces mesures hydrodynamiques ont été réalisées dans la phase de comblement du chenal de vidange. Les mesures bathymétriques indiquent que ce comblement s'effectue par le haut de plage. Ces observations suggèrent que :

- le déferlement plus intense en tête de chenal $(x \approx 14 \mathrm{~m})$ favorise le maintien de la barre ;

- un déferlement plus régulier au centre du bassin favorise le transport de sédiment vers la côte ;

- le courant de retour dans le chenal n'est pas assez fort pour contribuer notablement au transport de sédiment vers le large et compenser l'apport par le haut de plage.

Ces éléments contribuent à expliquer le comblement du chenal de vidange par migration de la barre transverse au centre du bassin et transport de sédiment par la circulation de haut de plage qui alimente le courant de retour dans le chenal.

\section{Conclusions}

Nous avons présenté dans cet article une modélisation physique de la formation et de la dégénérescence de chenaux de vidange, constituants la signature morphologique de courants sagittaux, lors d'une séquence accrétive. Ces résultats complètent la description des circulations par mesures Lagrangiennes réalisée par CASTELLE et al. (2010). Ces expériences ont par ailleurs permis de caractériser localement la dynamique du lit par mesures acoustiques et optiques (voir BERNI et al., 2010). Un réseau de capteurs de pression (majoritairement enfouis dans le sable) a aussi été déployé afin de mieux comprendre la dynamique couplée des ondes longues et de la zone de jet de rive. Le traitement de l'ensemble de ces données se poursuit. 

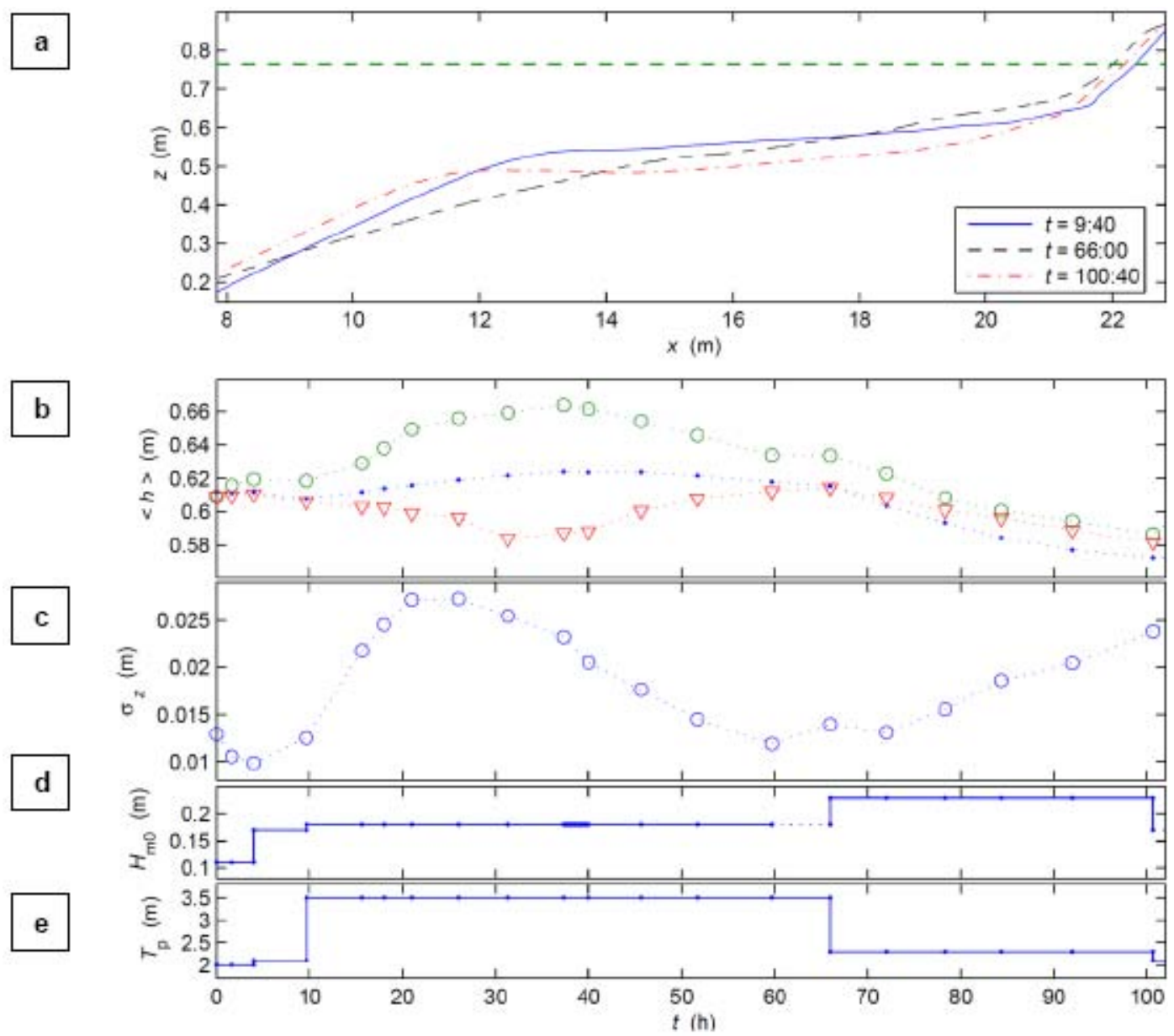

Figure 5. (a) Profils moyens: initial (-), fin de phase accrétive (- -) et fin de phase érosive (-.-). (b) Niveau moyen du lit (surface libre au repos en $\langle h\rangle=0.765 \mathrm{~m}$ ) pour le haut de plage $x \geq 14 \mathrm{~m}$ en $y=18.3 \mathrm{~m}(\mathrm{o})$, en $y=10.7 \mathrm{~m}(\nabla)$ et pour $y \leq 18.3 \mathrm{~m}(\cdot)$.

(c) Paramètre de tridimensionnalité. (d) Hauteur significative imposée aux batteurs. (e) période pic.
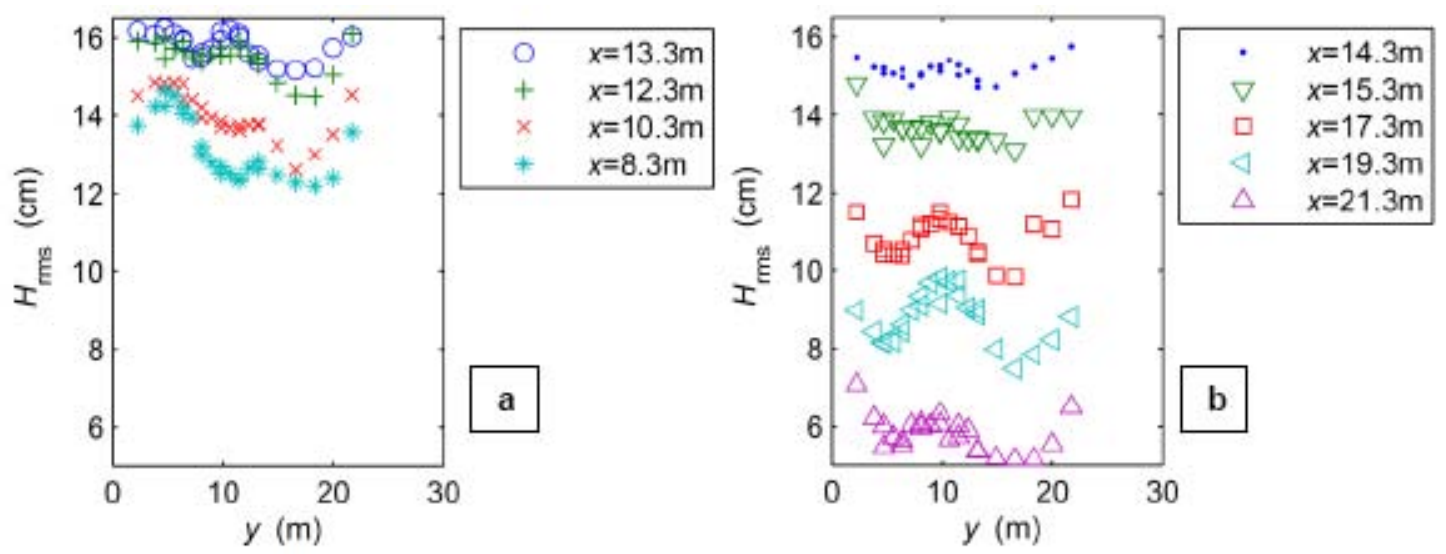

Figure 6. Variation longshore de la hauteur de vague quadratique moyenne pour différentes positions cross-shore (51:40 $\leq t \leq 59: 40)$. 

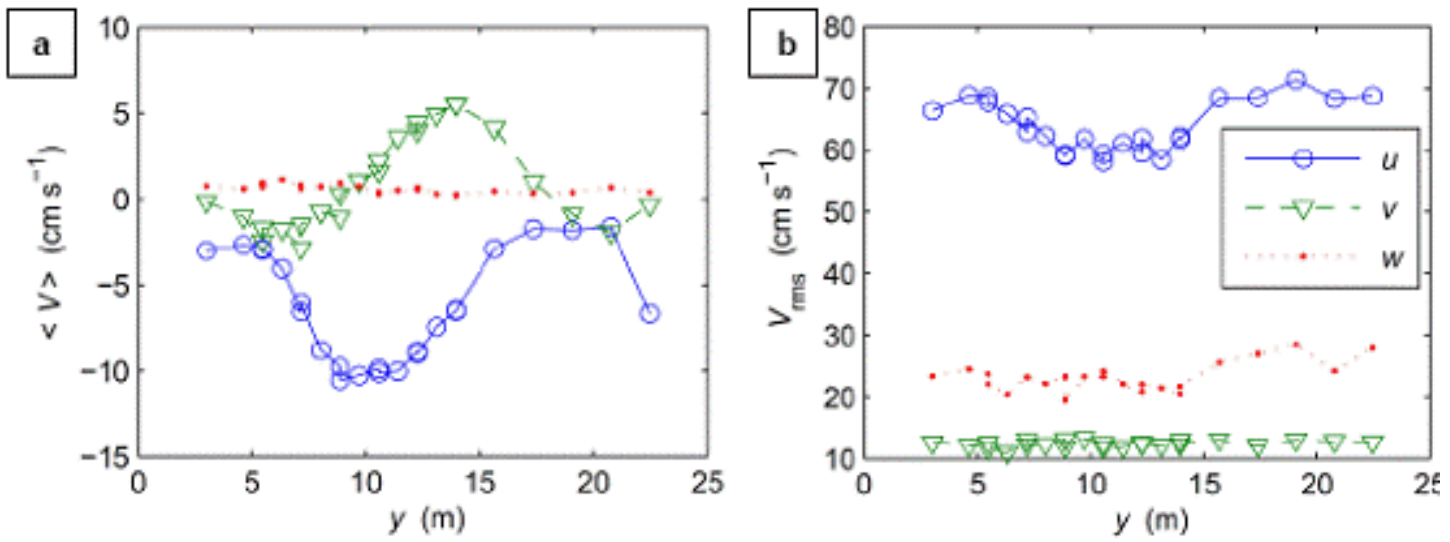

Figure 7. Variations longshore des vitesses moyennes (a) et des vitesses moyennes quadratiques (b). Composantes cross-shore (o), longshore ( $\nabla)$ et verticale (·) mesurées $6 \mathrm{~cm}$ au-dessus du lit en $x=15 \mathrm{~m}$.

\section{Remerciements}

Cette étude, menée dans le bassin du LHF (G-INP - SOGREAH), a été financée par l'ANR COPTER et le projet MODLIT (DGA/SHOM - INSU/RELIEFS). Le soutien technique de SOGREAH, en particulier G. Excoffier et L. Marcellin, a été fortement apprécié.

\section{Références bibliographiques}

BERNI C., MICHALLET H., BARTHELEMY E. (2010). Caractérisation de la dynamique du lit de sable sous l'action des vagues. $\mathrm{XI}^{\text {èmes }}$ Journées Nationales Génie Côtier - Génie Civil, Les Sables d'Olonne, pp 171-178. doi:10.5150/jngcgc.2010.021-B

BRUNEAU N., CASTELlE B., BONNETON P., PEDREROS R. (2009). Very Low Frequency motions of a rip current system: observations and modeling. J. of Coastal Res., SI 56, pp 1731-1735.

CASTELlE B., MiCHALLET H., MARIEU V., LECKLER F., DUBARDIER B., LAMBERT A., BERNI C., BARTHELEMY E., BOUCHETTE F., BONNETON P., KIMMOUN O., SOUS D., ALMAR R. (2010). Modélisation physique des courants d'arrachement : apport des mesures lagrangiennes. XI ${ }^{\mathrm{èmes}}$ Journées Nationales Génie Côtier - Génie Civil, Les Sables d'Olonne, pp 53-60. doi:10.5150/jngcgc.2010.007-C

GRASSO F., MICHALLET H., BARTHELEMY E., CERTAIN R. (2009). Physical modeling of intermediate cross-shore beach morphology: Transients and equilibrium states, J. Geophys. Res., 114, C09001. doi:10.1029/2009JC005308

MACMAHAN J.H., THORNTON E.B., RENIERS A.J.H.M. (2006). Rip current review. Coastal Engineering, 53, pp 191-208. doi:10.1016/j.coastaleng.2005.10.009

WRIGHT L.D., SHORT A.D. (1984). Morphodynamic variability of surf zones and beaches: A synthesis. Marine Geology, 56, pp 93-118. doi:10.1016/0025-3227(84)90008-2 